Article

\title{
Quantitative Descriptive Analysis of Traditional Herbal and Coffee Liqueurs Made with Grape Marc Spirit (Orujo)
}

\author{
Sandra Cortés-Diéguez ${ }^{1, *}$, Carmen Otero-Cerviño ${ }^{2}$, Hixinio Rodeiro-Mougán ${ }^{2}$ and \\ José Antonio Feijóo-Mateo ${ }^{2}$ \\ 1 Laboratory of Agro-food Biotechnology, CITI-Tecnópole, Parque Tecnológico de Galicia, \\ San Cibrao das Viñas, University of Vigo, 32900 Ourense, Spain \\ 2 CRIIGG of Spirits and Traditionally Liquours from Galicia, Pazo de Quián, \\ 15881 Sergude Boqueixón-A Coruña, Spain; carmenoteroc@gmail.com (C.O.-C.); \\ orujodegalicia@orujodegalicia.org (H.R.-M.); orujo@orujodegalicia.org (J.A.F.-M.) \\ * Correspondence: smcortes@uvigo.es; Tel.: +34-98-8387-416
}

Received: 11 May 2020; Accepted: 29 May 2020; Published: 5 June 2020

check for updates

\begin{abstract}
Orujo is a recognized traditional grape marc distillate from Galicia (NW of Spain). It is also employed as an alcohol base to elaborate coffee and herbal liqueurs and spirits. In this manuscript, quantitative descriptive analysis was applied to obtain the most important sensory terms that describe these traditional beverages. Thirteen trained panelists developed a complete sensory lexicon. Sixteen sensory descriptors (four in appearance, five in aroma, four in mouth, and three in aftertaste) were defined, valuated, and scored with the corresponding references, after elimination of hedonic, synonymous, and non-pertinent attributes according to statistical methods. The panelists evaluated a total of 464 samples in order to define their sensory profile. Panel performance was investigated showing good discriminatory ability, repeatability, and reproducibility. Principal Component Analysis (PCA) was also applied to identify the sensory descriptors that better discriminate the samples. The results obtained showed the importance of including new terms (orujo, chocolate-cocoa, floral, bitter, and astringent) in the tasting sheet, mainly in the case of coffee liqueurs to improve their sensory profile. The results of this study were useful for the development and implementation of an important tool for the corresponding regulating council in the sensory characterization and qualification of Galician liqueurs.
\end{abstract}

Keywords: spirits; coffee; herbal; liqueurs; panel training; principal component analysis; quantitative descriptive analysis; sensory analysis; taste evaluation form

\section{Introduction}

One of the most important logistical and environmental problems worldwide is the generation of waste from industries, mainly from the textile, livestock, agricultural, chemical, and wood sectors [1-3].

In wine-producing countries, the residues of winemaking, skins, pulp, seeds, and lees, far from being a problem, represent the opportunity to obtain a product with high added value like distillates or spirits, with the most popular being the Orujos from Spain, the Italian Grappas, the French Marcs, and the Portuguese Bagaçeiras [4-7]. All these distillates have in common that they come from of the distillation, under similar technology, of the winemaking residues once fermented. With a correct storage of the raw material and a proper distillation process [8-12], the spirits maintain the qualities of the grape varieties from which they come from and also the singular characteristics and authenticity of a traditional product linked to a certain area [13-15]. 
The majority of these distillates are consumed, after a dilution process to reduce the ethanol content $(40-45 \%(v / v))$, with or without aging time in oak barrels [16-18]. However, some of them are also flavored, such as Ouzos and Tsipouros [19-21], or used as an alcohol base to obtain liqueurs [22]. Liqueurs are sweet and alcoholic beverages obtained mixing different raw materials (plants, herbs, spices, seeds, flowers, fruits, coffee, dairy products) with distillates of agricultural origin [23]. It is also possible to distill fruit after alcoholic fermentation and then add sugar to obtain the corresponding beverage [24].

Natural or synthetic extracts are also used, which are not always allowed and whose presence detracts from the quality of the liqueur and, above all, tradition in its production. However, there are a wide variety of studies published on the volatile compounds provided by each herb and spice, making it possible to verify the presence of extracts in liqueurs, using CG-MS through not only the content of any compound but also in the relationship that can be established between them [25-28].

The liqueur elaboration process includes different stages, maceration, infusion, and distillation, or a mix of them. In these processes, volatile compounds, responsible for the aromatic notes, and phenolic compounds, with antioxidant properties and color, are transferred to the alcohol [29-32]. Phenolic compounds have a great influence on the quality of the liqueurs, mainly in the mouth due to their bitterness and astringency notes. These compounds are also responsible for the liqueur's appearance; however, the final color results from the addition of caramel, honey, or food-grade colorants.

Galicia (NW of Spain) is a traditional viticulture area that produces Orujo spirits from the distillation of winemaking residues. Since the grape varieties cultivated in this area are very aromatic (Albariño, Loureira, Treixadura, Godello, Mencía), the corresponding distillates have a lot of intense sensory descriptors, mainly defined by floral, vegetal, and fruity notes [33].

Besides the Orujo spirits, liqueurs made with aromatic plants, spices, and coffee are also traditionally elaborated in this area and highly valuated by their digestive and healthy properties, supporting such claims in the content of phenolic compounds and antioxidants present in the medicinal and aromatic plants used in the elaboration process [34]. According with this tradition, since 2004, coffee liqueurs and herbal liqueurs and spirits were included into the Geographical Indications of the Traditional Spirits and Liqueurs from Galicia (IGP) [35].

To protect their authenticity and establish a relationship with the geographic area and traditional practices in order to differentiate them from other similar products made in other areas, the IGP normative includes a concentration range for several chemical parameters and also a generic description of their sensory characteristics (Appendix A).

The raw materials allowed in the elaboration and generalities about the production process are fixed in the specifications of the Regulatory Council of the IGP. Mint, lemon verbena, aloysia, oregano, coriander, fennel, nutmeg, chamomile, rosemary, thyme, orange blossom, licorice, and cinnamon are considered the most traditional plants and species used to elaborate Galician liqueurs; however, production is allowed with any plant or species that is food grade. In all cases, herbal liqueurs must contain a minimum of three different plants and/or species.

In the case of coffee liqueur, it must be made with natural coffee, but no mention is made either of the species (Arabica or Robusta), the geographical origin, or the grain size (grounded or whole grain).

Taking into account the number and possible combinations of raw materials that can be used to elaborate these sweet and alcoholic beverages, the variety of existing liqueurs is very wide, showing different sensory characteristics, mainly in regard to aroma descriptors.

In the last years, several research works have been published regarding the optimal conditions of maceration, extraction of volatiles, characterization of raw materials, and volatile composition of Galician liqueurs [36]. These results allow very useful information to be obtained about the analytical composition of these traditional beverages to achieve their characterization and also identify the mainly phenolic and volatile compounds from plants, spices, coffee, and alcohol base responsible for their sensory profile. 
However, despite the singular composition of the liqueurs, since the introduction of these beverages in the IGP, liqueurs and young spirits have been sensory evaluated with the same taste evaluation form that was previously defined by quantitative descriptive analysis (QDA) application to young Orujo distillates [37]. Quantitative descriptive analysis is the more complete sensory tool applied in the food industry to describe and quantify sensory attributes [38].

The introduction of traditional liqueurs in the IGP implies that QDA must be used to train again the official panel in order to define a suitable liqueur lexicon to elaborate a specific tasting sheet in order to define the new characteristics, including adequate descriptors for the characterization and qualification of these alcoholic beverages. These results will be very useful to the producers and the technicians of the regulating council in the qualified sessions of liqueurs.

In this sense, the purposes of this study were (a) to develop and validate a complete liqueur lexicon using trained sensory panelists; (b) to determine the sensory attributes that are most important in the visual, aroma, taste, and aftertaste qualities to describe these traditional liqueurs in order to elaborate a useful tasting score sheet; and finally, (c) to define the sensory profile of these traditional beverages.

\section{Materials and Methods}

\subsection{Samples}

A total of 473 liqueurs and spirits were sensory evaluated at different stages of this study. All samples were collected by the technicians of the regulating council in several distilleries and producers sited in different areas from Galicia that produce these kinds of beverages according to the rules established into the legal normative of Geographic Indication Protected of Spirits and Traditional Liqueurs from Galicia. The samples were also analyzed to evaluate the concentration of the analytical parameters in order to their certification (Appendix A). Only those samples that met all the quality control requirements (sensorial and chemical) were included in this study.

Six samples of coffee and herbal liqueurs and spirits, two of each type, were evaluated in the first part of the quantitative descriptive analysis to generate the sensory descriptors in appearance, aroma, mouth, and aftertaste.

In the second part of QDA, three samples, one of each type, randomly chosen, were evaluated to validate the lexicon generated and validated by the training panelists and to familiarize them with the scale.

Finally, and with the new taste evaluation form defined by the panelists, 464 liqueurs were sensory evaluated from 2005 to 2019. In total, 213 samples belonged to the group of coffee liqueurs, 232 were herbal liqueurs, and 19 were herbal spirits.

\subsection{Sample Preparation}

All coffee and herbal liqueurs reported an ethanol concentration of $30 \%$ alcohol by volume and herbal spirits was on average $39.5 \%(v / v)$.

Samples $(20 \mathrm{~mL})$, without dilution, were served individually and coded with three-digit random numbers, in tulip spirit-taster glasses at $6-8{ }^{\circ} \mathrm{C}$. All sessions were developed in a professional tasting room composed of 20 independent tasting booths.

A plate with unsalted toasted bread and a bottle of natural water was given to each panelist to rinse the mouth between samples to clean the palate under similar conditions.

Sample presentation was randomized among all panelists during the sessions to define the sensory profile.

\subsection{Test Design}

The methodology applied in this research can be divided into three phases: Panel selection, panel training, and product testing using the QDA method. All training and sensory sessions were hosted by a panel leader and two technicians from the regulatory council as assistants. 


\subsubsection{Panel Selection and Pre-Training}

In total, 22 subjects, 13 males and 9 females, ranging in age from 31 to 55 years, all of them members of the official panel of "Geographic Indication Protected of the Spirits and Traditional Liqueurs from Galicia", participated in the sessions of the selection process. They had high experience in the sensorial analysis of traditional liqueurs and a special knowledge of this kind of alcoholic beverage, because most of them were distillers and local producers. However, to take part as a panelist in a quantitative descriptive analysis, training in the specific characteristics of the product being evaluated is necessary.

The development of the different tasks included in the pre-evaluation process was similar to that carried out for beer by Elgard et al., (2019) [39].

In the first session, a pre-screening questionnaire was completed by all subjects, including demographic information, sensory knowledge, involvement in other tasting panels, and availability for sensory training mainly for the future periodic product qualification sessions.

In order to evaluate the performance of each panelist, several pre-screening tests were applied before starting the training sessions. According to Ikes et al., (2017) [40], panelists were asked to carry out a basic taste test (sweet, acid, bitter, and salty) and also to identify odorants common to liqueurs and spirits. The basic taste test, with $10 \mathrm{~mL}$ of water solutions of each reference in plastic cups, was performed in the first day $(2 \mathrm{~h})$ of pre-screening training. Odor identification was evaluated in two 1-h sessions during two consecutive days. To carry out this test, 15 samples were prepared, 11 according to previous studies [37] and 4 by individual maceration, during one week of $10 \mathrm{~g} / \mathrm{L}$ of aromatic plants (chamomile, fennel and mint), $10 \mathrm{~g} / \mathrm{L}$ of spices (cinnamon and pepper), $40 \mathrm{~g} / \mathrm{L}$ of coffee (Arabica), and $40 \mathrm{~g} / \mathrm{L}$ of coffee (Robusta) prepared in an alcoholic solution at 30\% $(v / v)$. The 15 reference samples were served in 100-mL brown glass bottles with a screw top.

In the first session, the samples were smelled by the tasters and they were asked to write the descriptor that they perceived with more intensity. Each sample only could be smelt for a maximum of $30 \mathrm{~s}$. After $1 \mathrm{~h}$, the panelists had an hour break and then they repeated the test one more time. This test was to measure the ability to generate sensory descriptors.

In another session, on the next day, these 15 samples were smelled again, but in this test, the panelists were given a list with the name of the products and the main attributes associated with them. They were required to choose from this list the attribute that defined each sample. With this test, it was possible to evaluate the ability of the panelists to identify sensory descriptors in the different liqueur and spirit samples.

This pre-training process aimed to obtain information about the panelists and to evaluate their initial performance. A panelist had to achieve at least $80 \%$ acuity on the taste and odorant identification.

After the results obtained during the pre-training process were evaluated, only 13 subjects, 7 males and 6 females, ranging in age from 31 to 55 years, were selected to take part in the next steps of the QDA method.

\subsubsection{Lexicon Development and Training with References}

Lexicon development is one of the most important steps in sensory analysis [38] and an expert and trained panel must be carried out. Samples in this phase should be chosen to represent the product for sensory evaluation.

After the pre-training sessions, the 13 panelists selected were asked to freely develop, for around $1 \mathrm{~h}$ per sample, those terms that they considered that best described the liqueur and spirit samples. A representative collection of six samples (two herbal liqueurs, two coffee liqueurs, and two herbal spirits) were tested to identify the relevant appearance, aroma, mouth, and aftertaste attributes. These samples were evaluated over a total of three days. A great number of attributes in appearance, aroma, mouthfeel, and aftertaste were obtained. The total number of descriptors was higher in coffee liqueurs (87), mainly in aroma (29) and mouthfeel (26), due to the characteristics of this traditional beverage, different coffee origins, and other raw materials that can be added during the elaboration 
process (fruits, spiced, caramel, aromatic herbs). Besides, the sugar level in coffee liqueurs is usually higher than in herbal ones to balance the possible bitter and acid notes from coffee due to the presence of a high content of polyphenols [41]. The number of attributes generated for herbal spirits and herbal liqueurs were similar, 78 and 77, respectively, due to the only differences among both beverages being related to the analytical composition: Sugar level, alcohol content, and Orujo proportion (Appendix A).

Hedonic, redundant, synonyms, and inappropriate terms for descriptive analysis were then disregarded from the previous list, and the number of attributes was notably reduced [42]. In this sense, taking into account the particular characteristics of each beverage, a new list was created, including 5 terms in appearance, 20 in aroma, 11 in mouthfeel, and 14 in aftertaste. This list had too many attributes so, in order to reduce the number of sensory descriptors, the intensity (I) and frequency of citation (F) of each attribute were rated, and then, the geometric mean (GM) was calculated [43]. A summary of the sensory descriptors given by the expert panel, their geometric means, and classification is given in Table 1.

Table 1. Classification of the sensory descriptors by their geometric means.

\begin{tabular}{|c|c|c|c|c|c|}
\hline Descriptor & $M(\%) *$ & Class & Descriptor & $M(\%) *$ & Class \\
\hline Color Intensity & 30.4 & 4 & Spicy - Caustic & 24.2 & 4 \\
\hline Transparency & 50.5 & 3 & Sweet & 67.9 & 1 \\
\hline Clean & 24.3 & 5 & Soft & 8.9 & 8 \\
\hline Tonality and Shade & 53.0 & 2 & Light & 1.6 & 11 \\
\hline Aromatic plants (chamomile, coriander, rosemary, mint ... ) & 62.9 & 1 & Spicy-ardent & 17.7 & 6 \\
\hline Coffee & 26.3 & 3 & Alcoholic & 28.9 & 3 \\
\hline Fresh fruit (citric, stone fruit) & 22.4 & 4 & Drying & 2.6 & 10 \\
\hline Toasted-high-roast & 2.9 & 15 & Caustic & 4.7 & 9 \\
\hline Floral & 11.8 & 8 & \multicolumn{3}{|c|}{ Aftertaste } \\
\hline Vegetable/herbaceous & 18.3 & 6 & Aromatic plants & 32.5 & 2 \\
\hline Tobacco & 1.5 & 17 & Honey & 5.8 & 7 \\
\hline Orujo (Grape marc distillate) & 11.0 & 9 & Toasted-high-roast & 4.8 & 9 \\
\hline Spice (vanilla, cinnamon, & 23.6 & 5 & Orujo & 9.4 & 5 \\
\hline Balsamic & 7.0 & 10 & Impurity (mold, dirt) & 4.8 & 9 \\
\hline Dried fruit (nut, hazelnut) & 1.5 & 17 & Sulphur compounds & 2.4 & 12 \\
\hline Cocoa/chocolate & 5.1 & 12 & Burnt & 1.4 & 13 \\
\hline Persistence & 4.3 & 13 & Vegetal/herbaceous & 5.9 & 6 \\
\hline Hydrocarbon & 1.1 & 18 & Cocoa/chocolate & 4.3 & 10 \\
\hline Paper & 1.1 & 18 & Spice & 2.9 & 11 \\
\hline
\end{tabular}

${ }^{*} \mathrm{M}(\%)$ mean of geometric means.

Attributes with GM $>25 \%$ were selected to include them into the official taste evaluation form to describe the traditional liqueurs. However, by round-table discussion and consensus, the panel selected and refined the attributes that best described their perceptions and the panelist agreed to include terms like fruit in aroma, spicy-caustic in mouthfeel, and coffee in aftertaste, even though they had geometrical means lower than $25 \%$, due to their importance of these attributes to describe this kind of alcoholic and sweet beverages.

In accordance with these previous results, a new taste test evaluation form to certify liqueur samples was defined (Table 2). This taste test is divided into two columns, on the right shows the qualifying terms, the same as the original card, and on the left contains the descriptive terms, generated in the sensorial sessions by the panelists. It is necessary that a liqueur reaches 22 points in the total score of qualifying terms to obtain certification into Geographic Indication of the Spirits and Traditional Liqueurs from Galicia. 
Table 2. The final taste test evaluation form used in the sensory analysis of the herbal spirits, herbal liquors, and coffee liqueurs.

\begin{tabular}{|c|c|c|c|c|c|c|c|c|c|c|c|c|c|}
\hline \multicolumn{7}{|c|}{ Code Judge: } & \multicolumn{7}{|c|}{ Sample: } \\
\hline \multicolumn{7}{|c|}{ Descriptive Parameters } & \multicolumn{7}{|c|}{ Qualifying Parameters } \\
\hline $\begin{array}{l}\text { Appearance } \\
\text { Color Intensity }\end{array}$ & 0 & 1 & 2 & 3 & 4 & 5 & $\begin{array}{c}\text { Visual Phase } \\
\text { Quality }\end{array}$ & 0 & 1 & 2 & 3 & 4 & 5 \\
\hline $\begin{array}{c}\text { Tonality and Shade } \\
\text { Transparency } \\
\text { Brightness }\end{array}$ & & & & & & & & & & & & & \\
\hline Aroma & 0 & 1 & 2 & 3 & 4 & 5 & Aroma & 0 & 1 & 2 & 3 & 4 & 5 \\
\hline Intensity & & & & & & & Intensity & & & & & & \\
\hline Coffee & & & & & & & Fineness & & & & & & \\
\hline Aromatic herbs & & & & & & & Frankness & & & & & & \\
\hline \multicolumn{14}{|l|}{ Spiced } \\
\hline Fruity & & & & & & & & & & & & & \\
\hline Mouth & 0 & 1 & 2 & 3 & 4 & 5 & Mouth & 0 & 1 & 2 & 3 & 4 & 5 \\
\hline Sweet & & & & & & & Quality & & & & & & \\
\hline Dense greasy & & & & & & & Persistence & & & & & & \\
\hline Spicy & & & & & & & Fineness & & & & & & \\
\hline Alcoholic & & & & & & & Fragrance & & & & & & \\
\hline Aftertaste & 0 & 1 & 2 & 3 & 4 & 5 & General Impression & 0 & 1 & 2 & 3 & 4 & 5 \\
\hline Aromatic herbs & & & & & & & Harmony & & & & & & \\
\hline Coffee & & & & & & & Genuineness & & & & & & \\
\hline Persistence & & & & & & & Tot & sco & & & & & \\
\hline
\end{tabular}

When the terms to be used were agreed upon, the judges were trained, during 10 sessions, in the descriptors finally chosen using the corresponding references in aroma, mouth, and aftertaste. Table 3; Table 4 include a complete list of the attributes, definitions, and preparation procedures for each reference. During these sessions, the judges also were familiarized with the structured 6-point line scale, where $0=$ no detected, $1=$ weak, 2 = clear but no intense; 3 = intense note: 4 = high intense note; and $5=$ very high intense note.

Table 3. List of final attributes definitions, references, and references' preparation to train the panel.

\begin{tabular}{|c|c|c|c|}
\hline Descriptors & Definition & Reference & Preparation \\
\hline \multicolumn{4}{|l|}{ Appearance } \\
\hline Color Intensity & $\begin{array}{l}\text { Is the appearance that a } \\
\text { substance has as a result } \\
\text { of the way in which it } \\
\text { reflects light }\end{array}$ & - & - \\
\hline Tonality and Shade & $\begin{array}{c}\text { A type or degree of } \\
\text { a color }\end{array}$ & - & - \\
\hline Transparency & $\begin{array}{c}\text { Quality that a substance } \\
\text { has when you can see } \\
\text { through it }\end{array}$ & - & - \\
\hline Brightness & $\begin{array}{c}\text { A substance that is } \\
\text { shining strongly or is full } \\
\text { of light }\end{array}$ & - & - \\
\hline \multicolumn{4}{|l|}{ Aroma } \\
\hline Intensity & $\begin{array}{l}\text { Strength of the stimuli } \\
\text { perceived by the nose or } \\
\text { by olfactory receptors via } \\
\text { retronasal way }\end{array}$ & - & - \\
\hline
\end{tabular}


Table 3. Cont.

\begin{tabular}{|c|c|c|c|}
\hline Descriptors & Definition & Reference & Preparation \\
\hline Coffee & $\begin{array}{l}\text { Aroma associated } \\
\text { with coffee }\end{array}$ & $\begin{array}{l}\text { Natural coffee beans (Coffea } \\
\text { arabica and Coffea robusta) } \\
\text { from different origins: } \\
\text { Brazil, Colombia, Ethiopia, } \\
\text { Uganda and Vietnam }\end{array}$ & $\begin{array}{l}\text { For each type and origin, } \\
4 \mathrm{~g} \text { of coffee beans in } \\
100 \mathrm{~mL} \text { of ethanol/ } \mathrm{H}_{2} \mathrm{O} \\
30 \%(v / v) \text { solution. Serve } \\
\text { in } 100-\mathrm{mL} \text { brown glass } \\
\text { bottles with a screw top. }\end{array}$ \\
\hline Aromatic plants & $\begin{array}{l}\text { Aroma associated with } \\
\text { aromatic plants }\end{array}$ & $\begin{array}{l}\text { Dried natural plants, such } \\
\text { as: mint, aloysia, oregano, } \\
\text { coriander, fennel, } \\
\text { chamomile, rosemary, } \\
\text { thyme, orange blossom } \\
\text { and licorice }\end{array}$ & $\begin{array}{c}\text { For each plant, } 1 \mathrm{~g} \text { of } \\
\text { leaves, seeds or flowers } \\
\text { in } 100 \mathrm{~mL} \text { of } \\
\text { ethanol/ } \mathrm{H}_{2} \mathrm{O} 30 \%(\mathrm{v} / \mathrm{v}) \\
\text { solution. Serve in } \\
\text { 100-mL brown glass } \\
\text { bottles with a screw top. }\end{array}$ \\
\hline Spiced & $\begin{array}{l}\text { Aroma associated } \\
\text { with spices }\end{array}$ & $\begin{array}{l}\text { Ground nutmeg, dried } \\
\text { bark strip of cinnamon, } \\
\text { beans of vanilla and dried } \\
\text { flowers of clove }\end{array}$ & $\begin{array}{l}\text { Solutions of } 1 \mathrm{~g} \text { from } \\
\text { each spice in } 100 \mathrm{~mL} \text { of } \\
\text { ethanol/ } \mathrm{H}_{2} \mathrm{O} 30 \%(v / v) \\
\text { solution. Serve in } 100 \mathrm{~mL} \\
\text { brown glass bottles with } \\
\text { screw- top. }\end{array}$ \\
\hline \multirow[t]{2}{*}{ Fruity } & $\begin{array}{c}\text { Aroma associated } \\
\text { with fruit }\end{array}$ & Citrus (lemon, orange ... ) & $\begin{array}{l}\text { A drop of citral }(1 \mathrm{~g} / 100 \mathrm{~g} \\
\text { ethanol) on a cotton ball } \\
\text { into a glass bottle with a } \\
\text { screw top. }\end{array}$ \\
\hline & & Ripe (peach, apricot ... ) & $\begin{array}{l}\text { A drop of } \gamma \text {-Decalactone } \\
(1 \mathrm{~g} / 100 \mathrm{~g} \text { ethanol) on a } \\
\text { cotton ball into a glass } \\
\text { bottle with screw-top. }\end{array}$ \\
\hline \multicolumn{4}{|l|}{ Mouth } \\
\hline Sweet & $\begin{array}{l}\text { Sensations produced by } \\
\text { aqueous solutions of } \\
\text { several products such as } \\
\text { sucrose or fructose }\end{array}$ & Sucrose & $\begin{array}{l}\text { Solutions of sucrose } \\
(5-10 \mathrm{~g} / \mathrm{L}) \text { in water. Serve } \\
\text { in } 30-\mathrm{mL} \text { cups cover }\end{array}$ \\
\hline Dense-Unctuous & $\begin{array}{l}\text { Sensation produce in the } \\
\text { oral cavity related to } \\
\text { resistance to fluid }\end{array}$ & Condensed milk & $\begin{array}{l}1 \text { teaspoon of condensed } \\
\text { milk. Serve in } \\
\text { 30-mL cups. }\end{array}$ \\
\hline Spicy & $\begin{array}{c}\text { Trigeminal sensation in } \\
\text { mouth that produce heat } \\
\text { and piquancy similar to } \\
\text { eat pepper }\end{array}$ & Ground pepper & $\begin{array}{l}2 \mathrm{~g} \text { of ground pepper in } \\
100 \mathrm{~mL} \text { of ethanol/ } \mathrm{H}_{2} \mathrm{O} \\
30 \%(v / v) \text { solution. Serve } \\
\text { in } 100 \mathrm{~mL} \text { brown glass } \\
\text { bottles with screw- top. }\end{array}$ \\
\hline Alcoholic & $\begin{array}{l}\text { Burning sensation in the } \\
\text { mouth associated with } \\
40 \% \text { or greater alcohol }\end{array}$ & Grape marc distillate & $\begin{array}{l}100 \mathrm{~mL} \text { of grape marc } \\
\text { distillate at } 45 \%(v / v)\end{array}$ \\
\hline \multicolumn{4}{|l|}{ Aftertaste } \\
\hline Coffee & $\begin{array}{l}\text { Aftertaste associated } \\
\text { with coffee }\end{array}$ & $\begin{array}{l}\text { Dark roast coffee beans } \\
\quad(\text { Coffea arabica })\end{array}$ & $\begin{array}{l}2 \text { teaspoons of coffee, in } \\
\text { teabag, in } 250 \mathrm{~mL} \text { of } \\
\text { boiling water for } 5 \mathrm{~min} . \\
\text { Serve in } 30-\mathrm{mL} \text { cups } \\
\text { cover. }\end{array}$ \\
\hline Aromatic Plants & $\begin{array}{l}\text { Aftertaste associated } \\
\text { with aromatic plants }\end{array}$ & $\begin{array}{l}\text { A blend of aromatic plants, } \\
\text { such as mint, oregano, } \\
\text { fennel, thyme.... }\end{array}$ & $\begin{array}{l}3 \mathrm{~g} \text { of a blend of plants, } \\
\text { in teabag, in } 250 \mathrm{~mL} \text { of } \\
\text { boiling water for } 5 \mathrm{~min} \text {. } \\
\text { Serve in } 30-\mathrm{mL} \\
\text { cups cover }\end{array}$ \\
\hline
\end{tabular}


Table 3. Cont.

\begin{tabular}{cccc}
\hline Descriptors & Definition & Reference & Preparation \\
\hline Persistence & $\begin{array}{c}\text { Flavor sensation similar } \\
\text { to that which was } \\
\text { perceived whilst the } \\
\text { product was in the } \\
\text { mouth and while } \\
\text { continues for a } \\
\text { measurable period } \\
\text { of time }\end{array}$ & - & - \\
\hline
\end{tabular}

Table 4. Attributes selected to qualify the global quality in liqueurs and their corresponding definitions.

\begin{tabular}{|c|c|}
\hline Descriptors & Definition \\
\hline \multicolumn{2}{|l|}{ Appearance } \\
\hline - & Describe a product without particles and/or precipitates. Clean and with the expected color. \\
\hline Intensity & Describes the strength of the aroma \\
\hline Fineness & The quality of having delicate notes \\
\hline Frankness & Honest, sincere, a product without defects or strange aromas \\
\hline \multicolumn{2}{|r|}{ 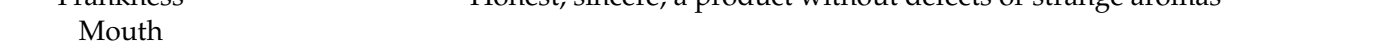 } \\
\hline Quality & Always in positive. Means how good is a product, to show a high standard \\
\hline Persistence & The quality of being persistent or longer in the time \\
\hline Fineness & The quality of being delicate \\
\hline Fragrance & A sweet or pleasant smell \\
\hline \multicolumn{2}{|l|}{ Global impression } \\
\hline Harmony & A pleasant sensation made by a good combination of different sensory perceptions \\
\hline Genuineness & $\begin{array}{l}\text { The quality of being real and exactly what it appears to be. A liqueur that show the sensory } \\
\text { characteristics that define these traditional beverages }\end{array}$ \\
\hline
\end{tabular}

\subsubsection{Sensory Profile}

Finally, 464 liqueur and spirit samples were sensorially evaluated with the QDA method using the descriptor list previously defined by the trained panelists. Sample presentation was randomized among all panelists that scored each sensory descriptor using the abovementioned 6-point-scale.

\subsubsection{Validation of the Panel}

Panel performance was evaluated by analysis of variance (ANOVA) in order to obtain reliable results on sensory analysis and to improve the selection and training of panelists. Using the sensory descriptors included in the taste test evaluation form (Table 3), the sources of variation analyzed were the samples, panelist, and session.

\subsubsection{Effect of Sample (Individual Discriminatory Ability)}

Thirteen sensory attributes, from the 16 evaluated, were significantly different, most of them at $99.9 \%$ of confidence $(p<0.0001)$. So, these 13 terms were the most important for describing the traditional liqueurs and for explaining their differential characteristics. The other three descriptors, aroma intensity, dense-greasy, and persistence, did not show significant differences among samples. These results suggest that differences between samples are small for these parameters, because liqueurs and spirits are very similar in global terms.

\subsubsection{Effect of Panelist (Panel Homogeneity)}

The effect panelist was only significant for persistence in aftertaste, $p<0.05$, reflecting the homogeneity of the panel to describe the samples with the lexicon defined and also the same interpretation of the structured scales. 


\subsubsection{Effect of Session (Individual Scoring Reproducibility)}

No significant differences between replicates in different sessions were found, which indicates that the panelist did not change the way they rated the liqueurs over time. They were capable of making reproducible judgments, i.e., the samples were evaluated in the same way in different sessions. So, the use of the attributes was consistent and reflected the reproducibility of the whole panel.

\subsection{Statistical Analysis}

All statistical analysis was performed using XLstat-Pro program for Windows (Addinsoft, New York, NY, USA). Two-way analysis of variance (ANOVA) was applied to establish whether significant difference ( $p \leq 0.05 ; p \leq 0.01 ; p \leq 0.001)$ existed. Class of liqueurs, judges, and repetition were considered as the factors. The multiple range tests (least-squares difference (LSD)) were applied to confirm the results obtained. Pearson's correlations between descriptive attributes and qualifying parameters were also calculated. Principal component analysis (PCA) was applied to attempt the separation of the three types of alcoholic beverage (coffee and herbal liqueurs and spirits) according to the descriptive and qualifying parameters.

\section{Results and Discussion}

This manuscript shows the first study on the quantitative descriptive analysis of traditional liqueurs and spirits made with Galician Orujo distillates, as an alcohol base, and aromatic herbs and coffee. Similar research works have been published by Donnell et al., (2007) [44] that applied sensory descriptive analysis to develop a list of descriptors for several distilled beverages and Caldeira et al., (2018) [45] that evaluated the sensory and analytical characteristics of blueberry liqueurs elaborated with wine and marc distillates.

In total, 464 samples (213 coffee liqueurs, 232 herbal liqueurs, and 19 herbal spirits) were sensory evaluated along 70 sessions from 2005 to 2019 with a maximum of 7 different samples per session. Liqueur and spirit samples were provided by different local manufacturers belonging to the Geographic Indication Protected of Spirits and Traditional Liqueurs from Galicia (IGP) and all of them were collected by technicians of the regulating council.

Initially, 22 panelists participated in previous selection sessions to evaluate their capacity in the sensory evaluation of distilled and liqueur beverages. Thirteen of them were selected to take part of this study. After several training sessions, during lexicon development, the panelist generated a total of 242 attributes to describe the coffee and herbal liqueurs and spirits. Finally, and after group discussion, 16 descriptors, 4 in appearance, 5 in aroma, 4 in mouth, and 3 in aftertaste, were identified by the panelists to be used in the sensory evaluation of these traditional beverages. All terms were defined, and the reference samples were selected, prepared, and scored. The new tasting sheet (Table 2) also included 10 qualifying parameters ( 1 in visual phase, 3 in aroma, 4 in mouth, and 2 to evaluate the general impression), which are the terms used till this moment by the official panel of the IGP, to qualify these beverages. Descriptive and qualifying parameters' intensities were scored on a 6-point scale (ranging from $0=$ no detected to $5=$ very high intense). By consensus between the regulatory council technicians and panelists, a liqueur and a spirit sample must obtain a total score $\geq 22$ in qualifying parameters to belong to the IGP and none of the individual qualifying parameters can be 0 (not detected). Taking into account the importance of qualifying terms in the sensory evaluation and their subjective definition (Table 4), it is very important evaluate the statistical correlation between qualifying and descriptive parameters.

\subsection{Pearson's Correlation between Descriptive and Qualifying Sensory Scores}

Pearson's correlations values $(r)$ between the scores obtained by descriptive and qualifying parameters in sensory testing of the spirit and liqueur samples are reported in Tables 5-7. 
Table 5. Pearson correlation values $(\mathrm{R})$ between descriptive and qualifying parameters in coffee liqueurs.

\begin{tabular}{|c|c|c|c|c|c|c|c|c|c|c|c|}
\hline Variables & $\begin{array}{l}\text { Visual } \\
\text { Quality }\end{array}$ & $\begin{array}{c}\text { Aroma } \\
\text { Intensity }\end{array}$ & $\begin{array}{c}\text { Aroma- } \\
\text { Fineness }\end{array}$ & $\begin{array}{c}\text { Aroma- } \\
\text { Frankness }\end{array}$ & $\begin{array}{l}\text { Mouth- } \\
\text { Quality }\end{array}$ & $\begin{array}{c}\text { Mouth- } \\
\text { Persistence }\end{array}$ & $\begin{array}{c}\text { Mouth- } \\
\text { Fineness }\end{array}$ & $\begin{array}{c}\text { Mouth- } \\
\text { Fragrance }\end{array}$ & Harmony & Genuineness & Score \\
\hline Color Intensity & 0.543 & 0.264 & 0.262 & 0.237 & 0.307 & 0.375 & 0.230 & 0.244 & 0.290 & 0.352 & 0.388 \\
\hline Tonality and Shade & 0.564 & 0.494 & 0.463 & 0.491 & 0.340 & 0.537 & 0.367 & 0.363 & 0.384 & 0.367 & 0.544 \\
\hline Transparency & 0.654 & 0.509 & 0.521 & 0.491 & 0.449 & 0.599 & 0.507 & 0.452 & 0.511 & 0.469 & 0.643 \\
\hline Brightness & 0.557 & 0.476 & 0.361 & 0.453 & 0.331 & 0.535 & 0.363 & 0.357 & 0.396 & 0.347 & 0.525 \\
\hline Intensity & 0.393 & 0.695 & 0.523 & 0.642 & 0.502 & 0.574 & 0.418 & 0.435 & 0.486 & 0.475 & 0.645 \\
\hline Aroma-Coffee & 0.257 & 0.427 & 0.361 & 0.463 & 0.411 & 0.382 & 0.365 & 0.376 & 0.458 & 0.485 & 0.501 \\
\hline Aroma-Aromatic herbs & 0.137 & 0.321 & 0.203 & 0.362 & 0.194 & 0.328 & 0.347 & 0.194 & 0.313 & 0.145 & 0.319 \\
\hline Aroma-Spiced & 0.236 & 0.500 & 0.312 & 0.426 & 0.377 & 0.450 & 0.456 & 0.295 & 0.424 & 0.295 & 0.473 \\
\hline Aroma-Fruity & 0.198 & 0.387 & 0.277 & 0.319 & 0.239 & 0.311 & 0.434 & 0.203 & 0.358 & 0.142 & 0.353 \\
\hline Sweet & 0.275 & 0.328 & 0.307 & 0.391 & 0.257 & 0.400 & 0.290 & 0.314 & 0.272 & 0.235 & 0.384 \\
\hline Dense-greasy & 0.266 & 0.183 & 0.224 & 0.232 & 0.254 & 0.187 & 0.297 & 0.121 & 0.326 & 0.276 & 0.295 \\
\hline Spicy & -0.060 & -0.042 & 0.047 & 0.083 & -0.065 & 0.101 & 0.096 & -0.092 & -0.085 & -0.100 & -0.017 \\
\hline Alcoholic & 0.425 & 0.322 & 0.276 & 0.297 & 0.245 & 0.404 & 0.333 & 0.197 & 0.288 & 0.284 & 0.381 \\
\hline Aftertaste-Coffee & 0.071 & 0.090 & 0.160 & 0.132 & 0.170 & 0.212 & 0.157 & 0.167 & 0.215 & 0.239 & 0.204 \\
\hline Aftertaste-Aromatic herbs & 0.206 & 0.332 & 0.322 & 0.299 & 0.331 & 0.468 & 0.372 & 0.230 & 0.394 & 0.295 & 0.405 \\
\hline Aftertaste-Persistence & 0.361 & 0.439 & 0.570 & 0.541 & 0.602 & 0.653 & 0.520 & 0.470 & 0.560 & 0.522 & 0.655 \\
\hline Score & 0.679 & 0.763 & 0.809 & 0.787 & 0.875 & 0.802 & 0.809 & 0.765 & 0.852 & 0.867 & 1.000 \\
\hline
\end{tabular}

Table 6. Pearson correlation values (R) between descriptive and qualifying parameters in herbal liqueurs.

\begin{tabular}{|c|c|c|c|c|c|c|c|c|c|c|c|}
\hline Variables & $\begin{array}{l}\text { Visual } \\
\text { Quality }\end{array}$ & $\begin{array}{c}\text { Aroma } \\
\text { Intensity }\end{array}$ & $\begin{array}{c}\text { Aroma } \\
\text { Fineness }\end{array}$ & $\begin{array}{c}\text { Aroma } \\
\text { Frankness }\end{array}$ & $\begin{array}{l}\text { Mouth } \\
\text { Quality }\end{array}$ & $\begin{array}{c}\text { Mouth } \\
\text { Persistence }\end{array}$ & $\begin{array}{c}\text { Mouth } \\
\text { Fineness }\end{array}$ & $\begin{array}{c}\text { Mouth } \\
\text { Fragrance }\end{array}$ & Harmony & Genuineness & Score \\
\hline Color Intensity & 0.630 & 0.311 & 0.457 & 0.267 & 0.250 & 0.213 & 0.236 & 0.291 & 0.311 & 0.284 & 0.380 \\
\hline Tonality and Shade & 0.676 & 0.495 & 0.546 & 0.466 & 0.406 & 0.437 & 0.354 & 0.414 & 0.431 & 0.440 & 0.547 \\
\hline Transparency & 0.826 & 0.477 & 0.416 & 0.396 & 0.323 & 0.345 & 0.322 & 0.417 & 0.448 & 0.354 & 0.505 \\
\hline Brightness & 0.641 & 0.507 & 0.400 & 0.365 & 0.369 & 0.351 & 0.393 & 0.447 & 0.426 & 0.386 & 0.499 \\
\hline Intensity & 0.454 & 0.453 & 0.258 & 0.307 & 0.199 & 0.309 & 0.053 & 0.256 & 0.209 & 0.150 & 0.308 \\
\hline Aroma-Aromatic herbs & 0.417 & 0.329 & 0.520 & 0.408 & 0.434 & 0.209 & 0.432 & 0.502 & 0.413 & 0.338 & 0.471 \\
\hline Aroma-Spiced & 0.360 & 0.198 & 0.375 & 0.353 & 0.392 & 0.259 & 0.346 & 0.410 & 0.296 & 0.303 & 0.385 \\
\hline Aroma-Fruity & 0.305 & 0.330 & 0.272 & 0.307 & 0.320 & 0.302 & 0.247 & 0.350 & 0.304 & 0.256 & 0.348 \\
\hline Sweet & 0.440 & 0.133 & 0.356 & 0.243 & 0.202 & 0.189 & 0.218 & 0.265 & 0.307 & 0.238 & 0.305 \\
\hline Dense-greasy & 0.373 & 0.162 & 0.402 & 0.374 & 0.378 & 0.315 & 0.361 & 0.413 & 0.448 & 0.382 & 0.424 \\
\hline Spicy & 0.024 & -0.061 & 0.136 & -0.006 & -0.069 & -0.002 & 0.043 & -0.110 & -0.140 & -0.091 & -0.031 \\
\hline Alcoholic & 0.240 & 0.292 & 0.272 & 0.259 & 0.276 & 0.371 & 0.145 & 0.261 & 0.208 & 0.283 & 0.304 \\
\hline Aftertaste-Aromatic herbs & 0.443 & 0.251 & 0.302 & 0.397 & 0.360 & 0.157 & 0.289 & 0.389 & 0.388 & 0.336 & 0.390 \\
\hline Aftertaste-Persistence & 0.452 & 0.331 & 0.290 & 0.483 & 0.411 & 0.330 & 0.213 & 0.478 & 0.441 & 0.387 & 0.448 \\
\hline Score & 0.612 & 0.759 & 0.847 & 0.934 & 0.921 & 0.824 & 0.850 & 0.914 & 0.942 & 0.932 & 1.000 \\
\hline
\end{tabular}

Pearson's correlation coefficients $r \geq 0.5$ are given in bold. 
Table 7. Pearson correlation values (R) between descriptive and qualifying parameters in herbal spirits.

\begin{tabular}{|c|c|c|c|c|c|c|c|c|c|c|c|}
\hline Variables & $\begin{array}{l}\text { Visual } \\
\text { Quality }\end{array}$ & $\begin{array}{c}\text { Aroma } \\
\text { Intensity }\end{array}$ & $\begin{array}{c}\text { Aroma } \\
\text { Fineness }\end{array}$ & $\begin{array}{c}\text { Aroma } \\
\text { Frankness }\end{array}$ & $\begin{array}{l}\text { Mouth } \\
\text { Quality }\end{array}$ & $\begin{array}{c}\text { Mouth } \\
\text { Persistence }\end{array}$ & $\begin{array}{l}\text { Mouth } \\
\text { Fineness }\end{array}$ & $\begin{array}{c}\text { Mouth } \\
\text { Fragrance }\end{array}$ & Harmony & Genuineness & Score \\
\hline Color Intensity & 0.723 & 0.645 & 0.543 & 0.387 & 0.258 & 0.224 & 0.241 & 0.241 & 0.241 & 0.258 & 0.422 \\
\hline Tonality and Shade & 0.741 & 0.602 & 0.482 & 0.741 & 0.582 & 0.642 & 0.576 & 0.609 & 0.609 & 0.582 & 0.695 \\
\hline Transparency & 0.526 & 0.298 & 0.227 & 0.512 & 0.359 & 0.444 & 0.367 & 0.398 & 0.398 & 0.359 & 0.437 \\
\hline Brightness & 0.526 & 0.298 & 0.227 & 0.512 & 0.359 & 0.444 & 0.367 & 0.398 & 0.398 & 0.359 & 0.437 \\
\hline Intensity & 0.679 & 0.484 & 0.306 & 0.655 & 0.436 & 0.567 & 0.407 & 0.543 & 0.543 & 0.436 & 0.568 \\
\hline Aroma-Aromatic herbs & 0.596 & 0.531 & 0.529 & 0.174 & 0.174 & 0.000 & 0.054 & 0.162 & 0.162 & 0.174 & 0.284 \\
\hline Aroma-Spiced & 0.465 & 0.342 & 0.319 & 0.650 & 0.455 & 0.563 & 0.505 & 0.465 & 0.465 & 0.455 & 0.531 \\
\hline Aroma-Fruity & 0.339 & 0.545 & 0.221 & 0.436 & 0.509 & 0.630 & 0.385 & 0.701 & 0.701 & 0.509 & 0.559 \\
\hline Sweet & 0.835 & 0.562 & 0.448 & 0.658 & 0.493 & 0.475 & 0.392 & 0.563 & 0.563 & 0.493 & 0.614 \\
\hline Dense-greasy & 0.747 & 0.424 & 0.459 & 0.546 & 0.436 & 0.378 & 0.475 & 0.339 & 0.339 & 0.436 & 0.515 \\
\hline Spicy & 0.637 & 0.033 & 0.197 & 0.361 & 0.000 & 0.000 & 0.112 & -0.112 & -0.112 & 0.000 & 0.124 \\
\hline Alcoholic & -0.068 & 0.666 & 0.561 & 0.327 & 0.655 & 0.567 & 0.611 & 0.611 & 0.611 & 0.655 & 0.594 \\
\hline Aftertaste Aromatic herbs & 0.548 & 0.604 & 0.654 & 0.415 & 0.311 & 0.180 & 0.226 & 0.290 & 0.290 & 0.311 & 0.433 \\
\hline Aftertaste Persistence & 0.628 & 0.612 & 0.538 & 0.846 & 0.611 & 0.732 & 0.658 & 0.628 & 0.628 & 0.611 & 0.736 \\
\hline Score & 0.446 & 0.912 & 0.891 & 0.908 & 0.968 & 0.909 & 0.935 & 0.923 & 0.923 & 0.968 & 1.000 \\
\hline
\end{tabular}

Pearson's correlation coefficients $r \geq 0.5$ are given in bold.




\subsubsection{Coffee Liqueurs}

The results in Table 5 show that all descriptor parameters, except spicy, were positively correlated with the qualifying parameters. The spicy note as a descriptor in liqueurs is mainly associated with bad quality of the distillate used as the alcohol base in the elaboration process. This perception is a consequence of the high presence of aldehydes or other volatile compounds from the first fraction of the distillate (compounds with high volatility), contributing herbaceous notes to the aroma and spicy notes in the mouth [46]. For this reason, it is justified that its presence in the liqueurs will have a negative impact in the global qualifying parameters. Visual quality showed high correlation values with all descriptive terms in appearance, mainly with transparency (0.654). This sensory descriptor was also highly correlated with the fineness, both in aroma (0.521) and in taste (0.507) and with taste persistence (0.599). This result shows the influence of subjectivity of the first view in the rest of the sensory parameters evaluated, and the importance of the visual appearance for consumer acceptance [47]. The descriptor intensity in the aroma was highly correlated with the intensity (0.695) and frankness (0.642), the corresponding qualifies' parameters. It is possible that the absence of off-flavor (frankness) allows the perception of a more intense coffee aroma in the liqueur. The tonality and shade (brown, mahogany) were positively correlated with taste persistence (0.537) so those coffee liqueurs with these visual attributes are more positively valuated in the mouth. Similar results were obtained by González et al. (2010) [48] in a sensory analysis of multifloral honey, and also found high correlation values between the color (visual) and terms used to describe the mouthfeel phase. Spiced notes (cinnamon, vanilla) showed a higher correlation value (0.500) with aroma intensity in coffee liqueurs, more than the individual coffee descriptor. The presence of spiced notes in these liqueurs was valuated well by the panelists increasing the total score. The sensory descriptor aftertaste persistence showed high correlation values with the majority of qualifying parameters in the aroma and in the mouth.

Visual parameters, global aroma intensity, coffee aroma, and aftertaste persistence are the descriptors that have greater influence in the global valuation of the coffee liqueurs. However, among the qualifying terms, those that are related to the authenticity of the beverage, genuineness (0.867), harmony (0.852), aroma fineness (0.809), and taste quality (0.875) have greater influence on the final score.

\subsubsection{Herbal Liqueurs}

Similar to in coffee liqueurs, only spicy notes showed a negative correlation value with all qualifying parameters (Table 6). The four sensory descriptors in the visual phase showed high correlation values with the visual quality, mainly the transparency $(0.826)$. Brightness was positively correlated with aroma intensity (0.507), whereas tonality and shade (0.546) and aromatic herbs $(0.520)$ were correlated with aroma fineness. Terms to describe the appearance are the first valuated by the panelist and can influence in the rest of the parameters. Herbal liqueurs with a high intense color (yellow, green, gold) are associated with more plants and spices used in the elaboration and thus a more intense aroma.

Among the sensory descriptors, only the tonality and shade (0.547) and transparency $(0.505)$ were correlated with the total score reached by the herbal liqueur samples, showing again the importance of the visual phase in the global impression. However, all qualifying parameters showed a high correlation value $(>0.8)$ with the total score, with visual quality $(0.612)$ and aroma intensity $(0.759)$ showing lower values.

\subsubsection{Herbal Spirits}

In the case of herbal spirits (Table 7), the majority of sensory descriptors showed high correlation values with the qualifying terms, mainly with visual quality, including the higher, sweet (0.835), and dense greasy (0.747). Spicy is a sensory descriptor that in herbal spirit showed a positive correlation value, mainly with visual quality (0.637). Spicy is related to the presence of Orujo in the sample, which is 
higher in herbal spirits than in liqueurs. Tonality and shade showed high correlation values with all the qualifying parameters and it was one of the most important in the global valuation (0.695). Aftertaste persistence was highly correlated with all qualifying parameters, mainly with taste persistence (0.732), and it was the descriptor that showed the higher correlation with the total score (0.736).

The singular characteristics of herbal spirits $(100 \%$ Orujo and sugar content lower than $100 \mathrm{~g} / \mathrm{L})$ imply that the term alcoholic has greater influence on the sensory quality than in the corresponding herbal liqueurs. So, this parameter showed a high positive correlation value with all qualifying descriptors, except with visual quality. All qualifying parameters showed high correlation values (>0.9) with the total score.

\subsection{Sensory Profile}

Quantitative descriptive analysis was used to compare the sensory profiles of the three types of traditional coffee and herbal liqueurs and spirits. The trained panel composed by 13 trained panelists assessed 454 samples, valuing each sensory descriptor using a 6-point-scale $(0=$ no detected, $1=$ weak, $2=$ clear but no intense; 3 = intense note: $4=$ high intense note; $5=$ very high intense note) (Table 2 ). The mean scores for each parameter were calculated and the results displayed in the form of spider diagrams (Figure 1).

During the sensory evaluation sessions, besides the 16 sensory descriptors included in the taste evaluation form, the panelists were free to add other attributes that they considered important to describe the sensory profile of the sample tasted. This term was scored in the column of descriptive parameters using the same 6-point scale. Taking into account the frequency and the intensity (score) of some of these terms, they were included to define the corresponding sensory profile. The new terms added for the three types of beverages evaluated were orujo in aroma, bitter and astringent in mouth and orujo, and fruity and spiced in after taste. Besides, in the case of herbal liqueurs and spirits, the descriptor "floral" was also included in aroma whereas in coffee liqueurs, terms like "caramel" and "chocolate-cocoa" were added to define the aroma and the aftertaste. Bitter and astringent are two descriptive terms also used to describe the flavor of citrus alcoholic beverages elaborated by the maceration of citrus peel in alcohol [49]. Both terms are associated with the presence of phenolic compounds in the liqueur that they were extracted from the coffee and the herbal plants during the maceration process in alcohol base (in this case Orujo) [50]. These bitter notes in some liqueurs are known, by their digestive effects, to stimulate the appetite or after a meal to aid food digestion [51].

The results in Figure 1 show that the visual parameters, mainly the tonality and shade and transparency, are very important to define herbal liqueurs and spirits, more than in coffee liqueurs due to their very high intensity brown color, which makes it difficult to evaluate the other parameters, mainly transparency. According to Castañeda-Olivares et al., (2010) [52], the visual appearance in beverages is an important determinant for consumer appearance, and is associated as an indicator of quality. The tonality and shade in herbal liqueurs and spirits depends on the plants used in the elaboration, on the ethanol concentration and titratable acidity of the alcohol base, and on the soaking time during the maceration process [53]. 

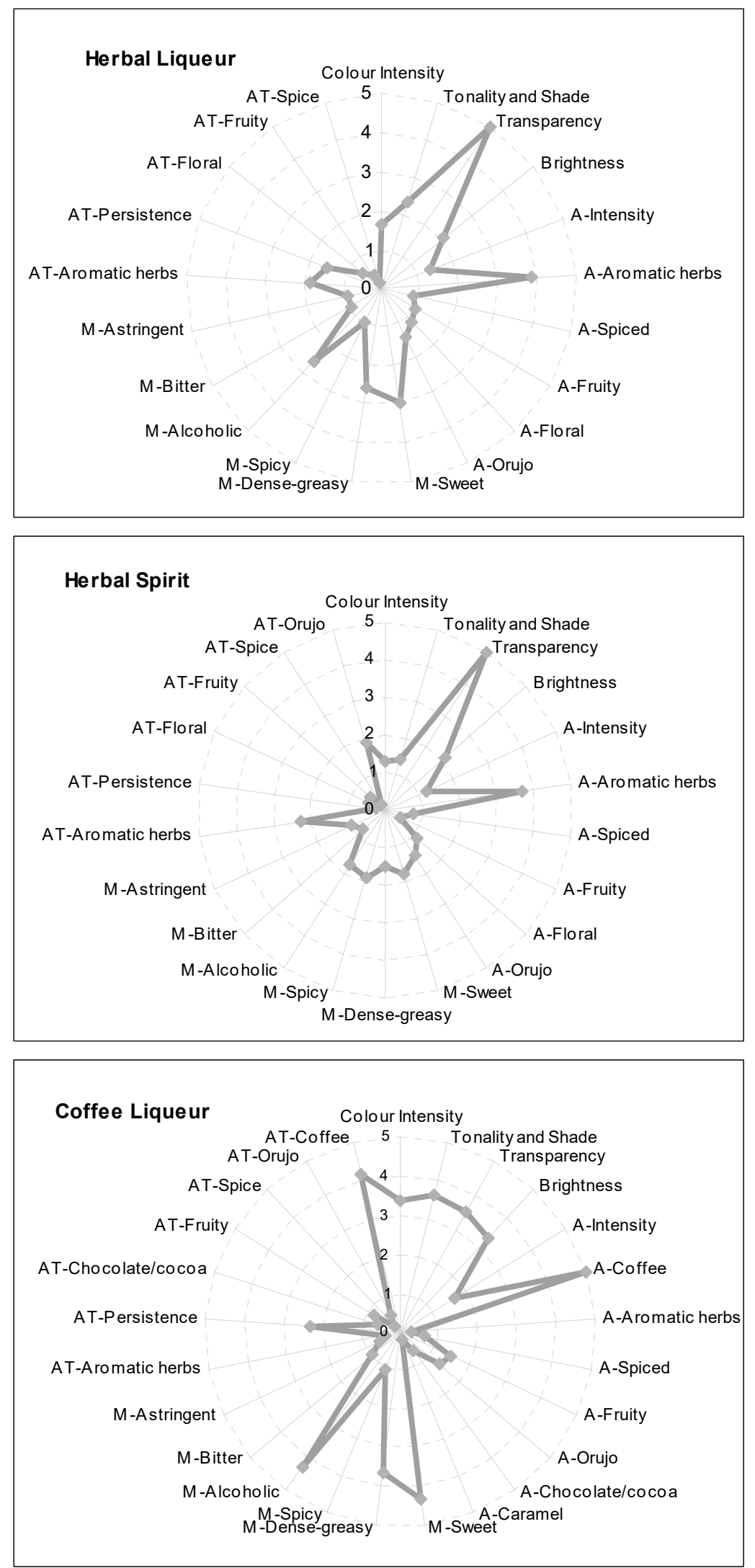

Figure 1. Sensory profile representing mean scores of the evaluated sensory attributes (A: aroma; AT: aftertaste; M: Mouth) for 232 herbal liqueurs; 19 herbal spirits and 213 coffee liqueurs. 
Herbal liqueurs and spirits are similar beverages with respect to the raw materials allowed (aromatic plants and spices) and to the elaboration process, but they are different in analytical composition (alcohol degree, sugar content, and \% of "Orujo"). For this reason, their sensory profile showed a very similar graph, but with some important differences. The herbal spirit profile was more complex than the herbal liqueurs in aroma and aftertaste; however, the descriptors in the mouth had a more intense score in herbal liqueurs, probably due to less alcohol and a greater sugar content. Herbal liqueurs and spirits also included, among their descriptors, Orujo (herbaceous, grape, green), which was not included in the first lexicon developed, but obviously it has great importance in the sensory quality of these traditional beverages. Orujo was the most important to describe the herbal spirits compared to the liqueur due to Orujo being $100 \%$ of the alcohol base used in the elaboration process. Floral notes were also more intense in herbal spirits. Probably, both descriptors, orujo and floral, could be due to the alcohol base taking into account that the grape varieties in Galicia have a great number and concentration of terpenes and C13-norisoprenoids [14]. Into the descriptor of aromatic herbs, the panelists included coriander, licorice, anise, mint, chamomile, and oregano, and in the spice notes, cinnamon and nutmeg; the score intensity for this attribute was similar in both beverages. The fruity note is mainly described as being like citric and orange, because the peel of this fruit is often used in the elaboration of these beverages. In the mouth, herbal liqueurs were more balanced, alcoholic notes are equilibrated with sweet and dense and greasy descriptors, whereas herbal spirits were more alcoholic, bitter, and astringent.

In the last years, herbal and coffee liqueurs have reduced the amount of Orujo in their elaboration to make the taste of the final product smoother and to increase the aromatic notes provided by the plants and the coffee, resulting in more complex profiles.

The sensory profile of coffee liqueurs was absolutely different than those the herbal liqueurs. Coffee liqueurs displayed a high intensity of coffee notes and included, with respect to the other two beverages, descriptors like chocolate cocoa and caramel that complete their sensory profile in aroma and in aftertaste. The contribution of fruity notes was very important for the direct aroma, mainly defined as being like orange peel due to orange being traditionally used to elaborate coffee liqueurs. The mouth profile was similar to the herbal liqueurs, but with a greater intensity, with a predominance of sweet and greasy notes, resulting in a balanced beverage. Traditionally, coffee liqueurs are sweeter than herbal liqueurs in order to reduce the bitter notes from the coffee. The term alcoholic in the mouth reached a higher score than in herbal liqueurs, despite them having a similar alcohol content.

The importance that some of these terms, floral, orujo, chocolate-cocoa, bitter, and astringent, have in the sensory profile suggests the need to propose the inclusion of these new descriptors to complete the tasting test evaluation form.

\subsection{Principal Component Analysis}

To interpret the results, principal component analysis was applied. PCA helps reduce the data and allows a better graphical representation of them to be obtained, making the interpretation of multivariate analysis easier. In this study, PCA was used to identify the sensory descriptors that best discriminated among the samples analyzed.

PCA was performed with those sensory descriptors that showed significantly different intensities between the samples. In the case of coffee liqueurs, only "aroma coffee" was the descriptor with a significant mean intensity score value between samples; thus, it was not possible to apply this statistical treatment to classify the 213 samples of coffee liqueur evaluated. In this sense, the results showed that while panelists were able to describe and qualify coffee liqueurs with the new taste test evaluation form, the selected descriptors were not adequate to discriminate among samples. This result means that probably, in this area, the coffee liqueurs have similar sensory characteristics, due to their elaboration process involving fewer ingredients (mainly coffee, orujo, and sugar) than herbal liqueurs and spirits. In addition, the sensory analysis of coffee is very complex with an abundant sensory lexicon to describe it [54]. These terms will also appear in coffee liqueurs with even greater intensity due to the extraction 
during the maceration process. For this reason, and taking into account the high number of samples evaluated, the results showed that it will be necessary to review the lexicon in order to include new and specific terms to describe coffee liqueurs applying a new QDA process.

\subsubsection{Herbal liqueurs}

A first PCA was performed on the score of the 12 sensory descriptive and qualifying parameters with significant differences (Figure 2A). The two first principal components, PC1 and PC2, accounted for $71.87 \%$ of the total variance $(57.92 \%$ and $13.95 \%$, respectively). This percentage of variance explained was higher than the value usually obtained in the sensory analysis of food [55].

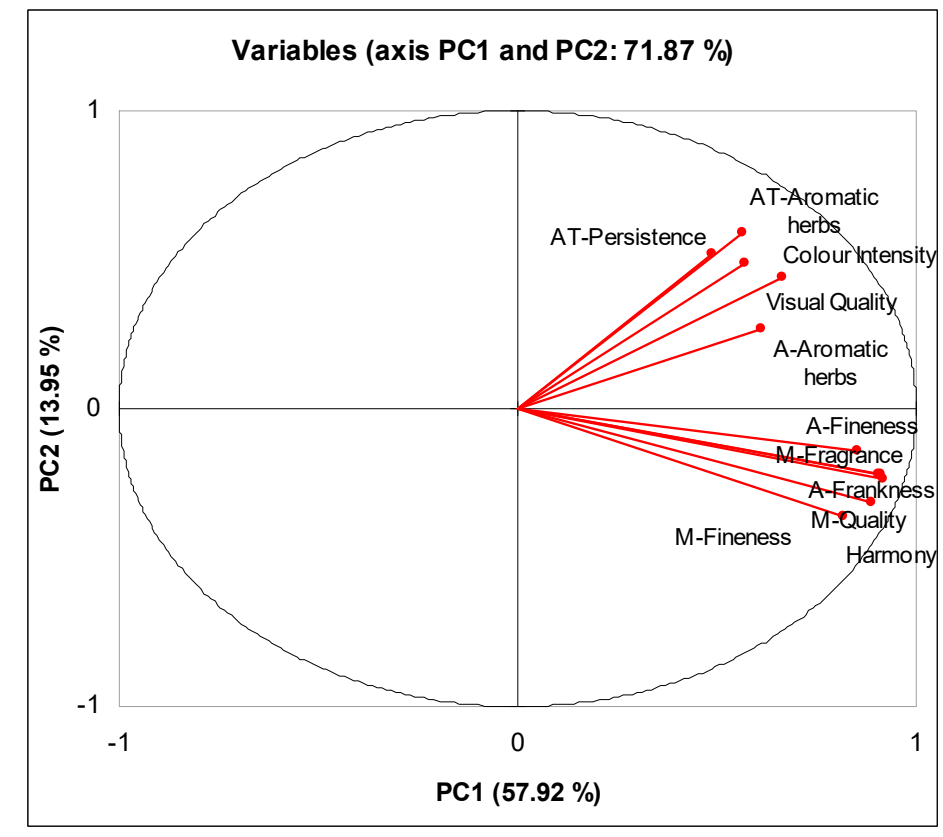

A

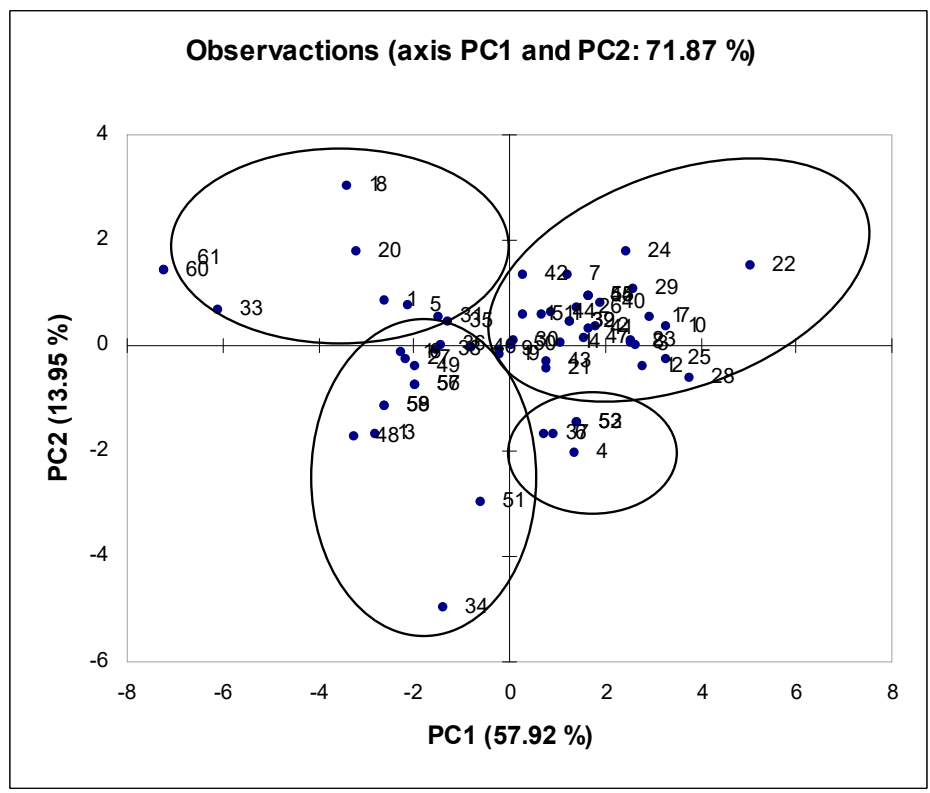

B

Figure 2. Component analysis score plot of (A) sensory descriptors and (B) samples (213 herbal liqueurs). A: aroma; AT: aftertaste; M: Mouth. 
The first component (PC1) was characterized by major and positive scores of the visual quality, aroma fineness, aroma frankness, mouth-quality, mouth-fineness, mouth-fragrance, and harmony, mainly qualifying terms. For the second principal component (PC2), the persistence in aftertaste showed the highest and positive value.

In Figure 2B, four groups of samples can be observed plotted on the plane defined by the two first principal components. The majority of samples were included in group 1 and sited in the positive side of PC1 and PC2. These samples were characterized by the high scores of the sensory qualifying and descriptive parameters. In contrast, the samples in group 2, the negative side of PC1 and PC2, were herbal liqueurs with a lower score in the qualifying parameters and a lower intensity in the descriptive attributes. Samples that were disqualified in the corresponding sensory sessions belonged to group 2. Samples included in group 3, the negative side of PC2 and the positive side of PC1, were liqueur samples that were valuated well in the qualification process despite obtaining lower scores in the descriptive parameters. This group is probably formed by liqueur samples with standard characteristics, i.e., no special attributes but without a sensory defect to decide to discard them in the qualification sessions. Only a small number of samples was included in group 4, with herbal liqueurs sited in the positive side of PC2 and negative side of PC1. This group of samples obtained a good score in persistence in aftertaste, but the rest of descriptive parameters (intensity of aromatic herbs in aroma and aftertaste, color, and visual quality) and qualified parameters were valuated badly. These samples were not qualified, probably mainly due to a defect in the visual quality (turbidity or artificial color), taking into account the importance of this phase in the global qualification score.

\subsubsection{Herbal Spirits}

All parameters showed significant differences between the herbal spirits, so the first PCA was performed on the score of the total 24 descriptive and qualifying parameters included in the taste test evaluation form. The two first principal components, PC1 and PC2, accounted for $75.75 \%$ of the total variance $(54.29 \%$ and $21.47 \%$, respectively) (Figure $3 \mathrm{~A}$ ).

The first component (PC1) was positively correlated with the eight qualifying parameters included in the taste sheet and with the majority of the sensory descriptors, visual (tonality and shade, transparency, and brightness), aroma (intensity, spiced, and fruity), mouth (sweet, dense-greasy), and aftertaste (persistence). The second principal component (PC2) was positively correlated with alcoholic notes and negatively correlated with spicy.

The plot of Figure 3B shows a good separation of herbal spirits in four groups. The majority of samples (47\%) were sited on the positive side of PC1 and PC2 and characterized by high scores of the sensory qualifying and descriptive parameters; they were the best valuated herbal liqueurs. Only two samples were included in group 2, the positive side of PC2 and negative side of PC1, characterized by alcoholic notes in the aroma. The majority of herbal spirits contain around $37.5 \%(v / v)$ of ethanol, but it is allowed reach $50 \%(v / v)$, so it is probably that those two samples in group 2 were herbal spirits with a high alcohol content that can reduce the perception of the other aromatic attributes, and for this reason, they were negatively correlated with the rest of sensory descriptors and with all qualifying parameters. Four samples were included in group 3, the negative side of PC1 and PC2, and they were positively correlated with spicy notes and negatively correlated with the rest of the attributes. These herbal spirits were probably elaborated with grape marc distillate (Orujo) with a high content of $\mathrm{C} 6$ compounds and aldehydes, defined by herbaceous and green notes. Four herbal spirits were belonged to group 4, the positive side of PC1 and negative side of PC2. These samples were positively correlated with the majority of descriptive parameters, mainly with those relative to the visual quality (transparency, tonality, and shade), with aroma (spiced, fruity, intensity) and mainly with those terms relative to attributes in the mouth (sweet, dense-greasy). 


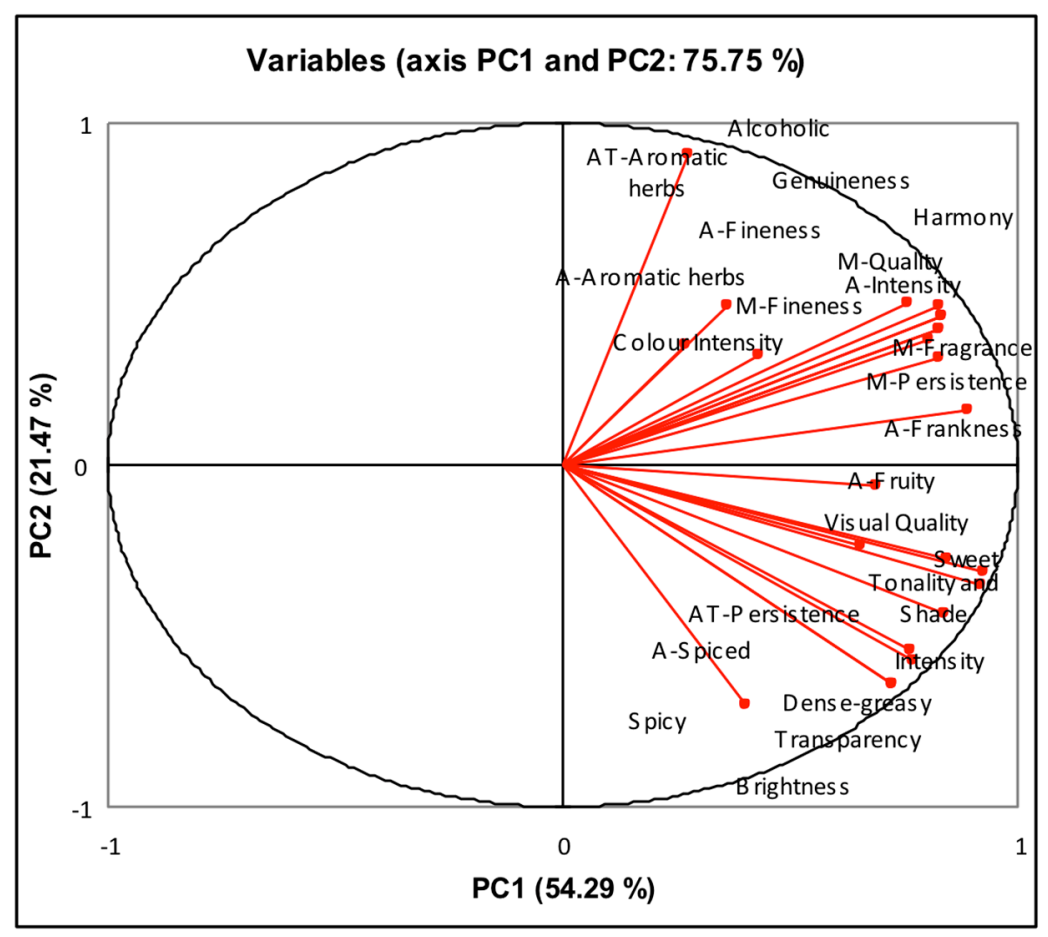

A

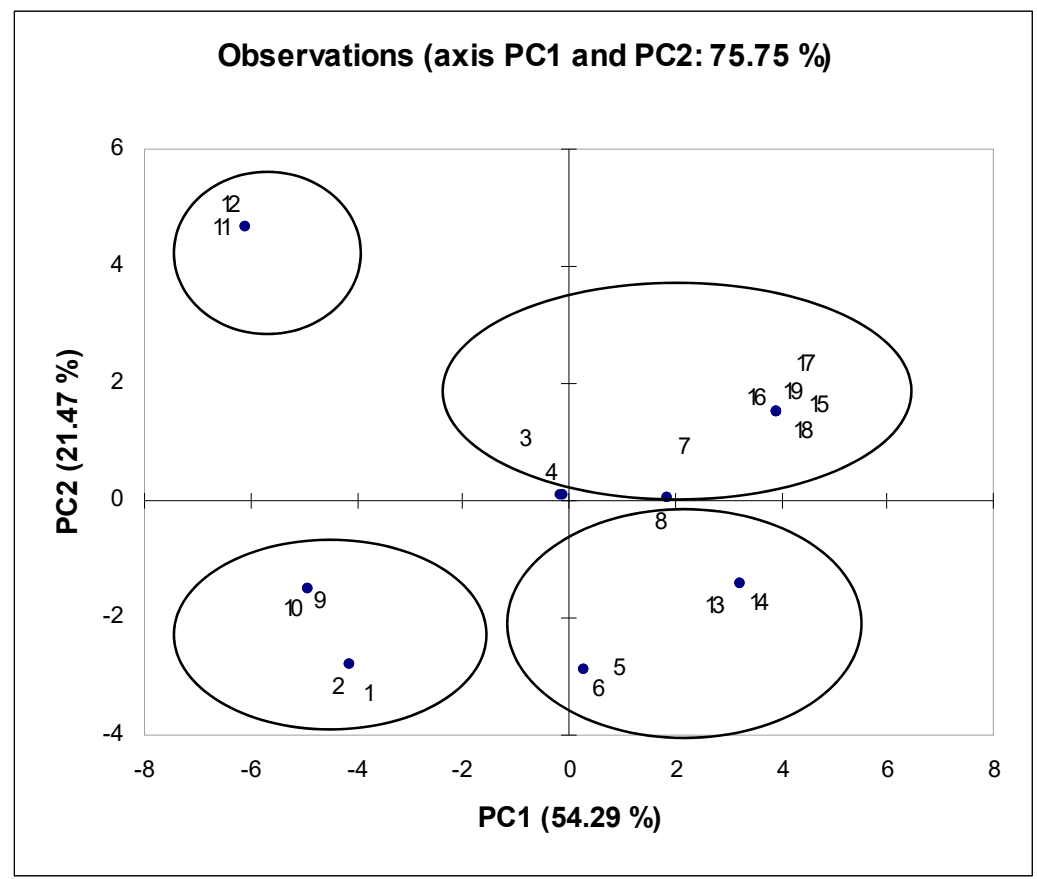

B

Figure 3. Principal component analysis score plot of (A) sensory descriptors and (B) samples (19 herbal spirits); A: aroma; AT: aftertaste; M: Mouth.

\section{Conclusions}

The inclusion of traditional coffee and herbal liqueurs and spirits in the Geographic Indication Protected of Spirits and Traditional Liqueurs from Galicia make necessary to have a tasting test evaluation form for their sensory description by the official tasting panel. In this sense, this study 
described the uses of quantitative descriptive analysis to define and validate a suitable lexicon for describing the sensory characteristics, in appearance, aroma, mouth, and aftertaste, of herbal and coffee liqueurs and spirits elaborated with Orujo (grape marc spirit from Galicia). After discarding the hedonic, synonymous, redundant, and non-pertinent attributes according to statistical methods, 13 trained panelists developed a complete lexicon, including descriptors, definitions, and references. A total of 16 sensory descriptors were approved by the panel, 4 in appearance ("color intensity", "tonality and shade", "transparency", and "brightness"), 5 in aroma ("aroma intensity", "coffee", "aromatic herbs", "spiced", and "fruit"), 4 in mouth ("sweet", "dense-greasy", "spicy", and "alcoholic"), and 3 in aftertaste ("aromatic herbs", "coffee", and "persistence"). All of them were scored using a 6-point-scale. After sensory evaluation of 464 samples (213 coffee liqueurs, 232 herbal liqueurs, and 19 herbal spirits), during 70 sessions from 2005 to 2019, the mean score for each descriptor was used to define the corresponding profile. The panel performance was also evaluated, and shown to have good discriminatory ability, repeatability, and reproducibility. The list of 16 descriptive terms was completed with other sensory descriptors like "floral" and "Orujo" and "chocolate-cocoa" and "caramel" in the aroma of herbal and coffee liqueurs, respectively, and "bitter" and "astringent" as descriptors in the mouth. These last terms were freely added, by the panelist, to the list of descriptors along the sensory sessions. PCA analysis was applied to identify the sensory descriptors that better discriminated the samples. Results showed that, in the case of coffee liqueurs, it is necessary to revise the lexicon employed to include more specific descriptive parameters for this product, taking into account that only the coffee aroma show significant differences among samples. In summary, the results obtained in this research help panelists and regulatory council technicians to describe and qualify coffee and herbal liqueurs and spirits. The results can also be extrapolated to other areas that produce similar beverages.

Author Contributions: Conceptualization, S.C.-D., C.O.-C., H.R.-M., and J.A.F.-M.; Methodology, S.C.-D., C.O.-C., H.R.-M., and J.A.F.-M.; Software, S.C.-D., C.O.-C., H.R.-M., and J.A.F.-M.; Validation, S.C.-D., C.O.-C., H.R.-M., and J.A.F.-M.; Formal analysis, S.C.-D., C.O.-C. and H.R.-M.; Investigation, S.C.-D., C.O.-C., H.R.-M., and J.A.F.-M.; Resources, S.C.-D., C.O.-C., H.R.-M., and J.A.F.-M.; Data curation, S.C.-D.; Writing-original draft preparation, S.C.-D.; Writing-review and editing, S.C.-D.; Visualization and Supervision, all authors. All authors have read and agreed to the published version of the manuscript.

Funding: This research received no external funding.

Conflicts of Interest: The authors declare no conflict of interest.

\section{Appendix A}

Table A1. Quality control requirements (chemical and sensorial) established for the three types of beverages, according to the Regulating Council rules.

\begin{tabular}{|c|c|c|c|c|c|c|}
\hline & \multicolumn{2}{|c|}{ Herbal Spirit } & \multicolumn{2}{|c|}{ Herbal Liqueur } & \multicolumn{2}{|c|}{ Coffee Liqueur } \\
\hline & Maximun & Minimum & Maximun & Minimum & Maximun & Minimum \\
\hline Alcohol degree $(\% v / v)$ & 50 & 37.5 & 40 & 20 & 40 & 20 \\
\hline Methanol (g HL ${ }^{-1}$ a.a.) ${ }^{1}$ & 950 & 200 & 950 & 50 & 950 & 50 \\
\hline Total acidity (in acetic acid, $\mathrm{g} \mathrm{HL}^{-1}$ a.a.) & 150 & - & 150 & - & - & - \\
\hline Acetaldehyde ( $\mathrm{g} \mathrm{HL}^{-1}$ a.a.) & 150 & - & 150 & - & 150 & - \\
\hline Ethyl acetate (g HL ${ }^{-1}$ a.a.) & 250 & - & 250 & - & 250 & - \\
\hline Higher alcohols ${ }^{2}$ ( g HL $^{-1}$ a.a.) & 600 & 225 & 600 & 60 & 600 & 60 \\
\hline $\mathrm{Cu}\left(\mathrm{mg} \mathrm{L}^{-1}\right)$ & 9 & - & 9 & - & 9 & - \\
\hline Total sugars $\left(\mathrm{gL}^{-1}\right)$ & 100 & - & - & 100 & - & 100 \\
\hline Appearance & \multicolumn{2}{|c|}{$\begin{array}{l}\text { Translucent, clean, with } \\
\text { colour from yellow pale } \\
\text { to green }\end{array}$} & \multicolumn{2}{|c|}{$\begin{array}{l}\text { Translucent, clean, with } \\
\text { colour from yellow pale } \\
\text { to green }\end{array}$} & \multicolumn{2}{|c|}{$\begin{array}{c}\text { Clean, with colour from } \\
\text { roasted amber to roasted } \\
\text { mahogany }\end{array}$} \\
\hline Aroma & \multicolumn{2}{|c|}{$\begin{array}{l}\text { Intense, fine, delicate } \\
\text { with floral notes, high } \\
\text { intensity of Orujo, } \\
\text { absence of musty smell, } \\
\text { burning, dirt and } \\
\text { acetic notes }\end{array}$} & \multicolumn{2}{|c|}{$\begin{array}{l}\text { Intense, fine, delicate } \\
\text { with floral notes, low } \\
\text { intensity of Orujo, } \\
\text { balsamic, aromatic herbs, } \\
\text { absence of moisture, } \\
\text { burning, dirt and } \\
\text { acetic notes }\end{array}$} & \multicolumn{2}{|c|}{$\begin{array}{l}\text { Intense, fine, delicate } \\
\text { with hints of freshly } \\
\text { roasted coffee, cocoa and } \\
\text { chocolate, bottom of a } \\
\text { distilled spirits of fine } \\
\text { and elegant Orujo }\end{array}$} \\
\hline
\end{tabular}


Table A1. Cont.

\begin{tabular}{|c|c|c|c|}
\hline & Herbal Spirit & Herbal Liqueur & Coffee Liqueur \\
\hline & Maximun Minimum & Maximun Minimum & Maximun Minimum \\
\hline Mouthfeel & $\begin{array}{l}\text { Persistent, balanced, } \\
\text { no alcoholic }\end{array}$ & Sweet & $\begin{array}{l}\text { Sweet, dense, enveloping, } \\
\text { creamy, persistent. It can } \\
\text { also present a bitter but } \\
\text { elegant nuance }\end{array}$ \\
\hline Aftertaste & $\begin{array}{l}\text { Floral, aromatic herbs } \\
\text { and spices notes }\end{array}$ & $\begin{array}{l}\text { Aromatic herbs and } \\
\text { balsamic notes }\end{array}$ & $\begin{array}{c}\text { Coffee and caramel notes, } \\
\text { and subtle notes of } \\
\text { chocolate or cocoa }\end{array}$ \\
\hline
\end{tabular}

\section{References}

1. Flores, R.A.; Shanklin, C.W.; Loza-Garay, M.; Wie, S.H. Quantification and characterization of food processing wastes/residues. Compos. Sci. Util. 1999, 7, 63-71. [CrossRef]

2. Münster, M.; Ravn, H.; Hedegaard, K.; Juul, N.; Ljunggren Söderman, M. Economic and environmental optimization of waste treatment. Waste Manag. 2015, 38, 486-495. [CrossRef]

3. Ahamed, A.; Yin, K.; Ng, B.J.H.; Ren, F.; Chang, V.W.-C.; Wang, J.-Y. Life cycle assessment of the present and proposed food waste management technologies from environmental and economic impact perspectives. J. Clean. Prod. 2016, 131, 607-614. [CrossRef]

4. ECC. Council Regulation; 1576/89; Official Journal of the European Communities: Brussels, Belgium, 1989; Volume L160, pp. 1-17.

5. Da Porto, C. Grappa and grape-spirit production. Crit. Rev. Biotech. 1998, 18, 13-24. [CrossRef]

6. Silva, M.L.; Macedo, A.C.; Malcata, F.X. Review: Steam distilled spirits from fermented grape pomace. Food Sci. Technol. Int. 2000, 6, 285-300. [CrossRef]

7. Cortés, S.; Rodríguez-Solana, R.; Salgado, J.M.; Dominguez, J.M. Comparative study between Italian and Spanish grape marc spirits in terms of major volatile compounds. Food Control. 2011, 22, 673-680. [CrossRef]

8. Cortés, S.; Gil, L.; Fernández, E. Concentration of volatiles in marc distillates from Galicia according to storage conditions of the grape pomace. Chromatographia 2001, 53, 406-411. [CrossRef]

9. Cortés, S.; Rodríguez, J.M.; Salgado, J.M.; Domínguez, J.M. The storage of grape marc: Limiting factor in the quality of the distillate. Food Control. 2010, 21, 1545-1549. [CrossRef]

10. Da Porto, C. Volatile composition of 'grappa low wines' using different methods and conditions of storage on an industrial scale. Int. J. Food Sci. Technol. 2002, 37, 395-402. [CrossRef]

11. Da Porto, C.; Decorti, D. Effect of cooling conditions on separation of volatile compounds in grappa using tray and packed columns without reflux. Int. J. Food Sci. Technol. 2008, 43, 638-643. [CrossRef]

12. Silva, L.; Malcata, X. Effects of time of grape pomace fermentation and distillation cuts on the chemical composition of grape marcs. Z. Lebens. Unters A 1999, 208, 134-143. [CrossRef]

13. Milicevic, B.; Banovic, M.; Kovacevic-Ganic, K.; Gracin, L. Impact of grape varieties on wine distillates flavour. Food Technol. Biotech. 2002, 40, 227-232.

14. Diéguez, S.; de la Peña, M.L.; Gómez, E. Approaches to spirit aroma: Contribution of some aromatic compounds to the primary aroma in samples of Orujo spirits. J. Agric. Food Chem. 2003, 51, 7385-7390. [CrossRef] [PubMed]

15. Gerogiannaki-Christopoulou, M.; Kyriakidis, N.V.; Panagiotis, E.A. Effect of grape variety (Vitis vinifera L.) and grape pomace fermentation conditions on some volatile compounds of the produced grape pomace distillate. J. Int. Sci. Vigne Vin. 2004, 38, 225-230. [CrossRef]

16. De Rosso, M.; Cancian, D.; Panighel, A.; Dalla Vedova, A.; Flamini, R. Chemical compounds released from five different woods used to make barrels for aging wines and spirits: Volatile compounds and polyphenols. Wood Sci. Technol. 2009, 43, 375-385. [CrossRef]

17. Canas, S.; Belchior, A.P.; Spranger, M.I.; Bruno-de-Sousa, R. HPLC method for the quantification of phenolic acids, phenolic aldehydes, coumarins and furanic derivatives in different kinds of toasted wood used for the ageing of brandies. Anal. Meth. 2011, 3, 186-191. [CrossRef] 
18. Rodríguez-Solana, R.; Rodríguez, N.; Dominguez, J.M.; Cortés, S. Characterization by chemical and sensory analysis of comercial grape marc distillate (Orujo) aged in oak wood. J. Inst. Brew. 2012, 118, 205-212. [CrossRef]

19. Soufleros, E.H.; Mygdalia, S.A.; Natskoulis, P. Characterization and safety evaluation of the traditional Greek fruit distillate "Mouro" by flavour compounds and mineral analysis. Food Chem. 2004, 86, 625-636. [CrossRef]

20. Karapanagioti, H.K.; Bekatorou, A. Alcohol and dilution water characteristics in distilled anis (Ouzo). J. Agric. Food Chem. 2014, 62, 4932-4937. [CrossRef]

21. Vlontzos, G.; Niavis, S.; Duquenne, M.N. Introduction on the market of Tsipouro a greek traditional liquor precursor of Ouzo. Int. J. Eur. Med. Stud. 2014, 7, 175-188.

22. Sliwinska, M.; Wisniewska, P.; Dymerki, T.; Wardencki, W.; Namiensnik, J. The flavour of fruit spirits and fruit liqueurs: A review. Flavour Frag. J. 2015, 30, 197-207. [CrossRef]

23. Satora, P.; Tuszynski, T. Chemical characteristics of Sliwowica Lacka and other plum brandies. J. Sci. Food Agric. 2008, 88, 167-174. [CrossRef]

24. Iscan, G.; Kirimer, N.; Kürkcüoglu, M.; Baser, H.C.; Demirci, F. Antimicrobial screening of Mentha piperita essential oils. J. Agric. Food Chem. 2002, 50, 3943-3946. [CrossRef] [PubMed]

25. Flamini, G.; Cioni, P.L.; Morelli, I.; Macchia, M.; Ceccarini, L. Main agronomic-productive characteristics of two ecotypes of Rosmarinus officinalis L. and chemical composition of their essential Oils. J. Agric. Food Chem. 2002, 50, 3512-3517. [CrossRef]

26. Gachkar, L.; Yadegari, D.; Rezaei, M.B.; Taghizadeh, M.; Astaneh, S.A.; Rasooli, I. Chemical and biological characteristics of Cuminum cyminum and Rosmarinus officinalis essential oils. Food Chem. 2007, 102, 898-904. [CrossRef]

27. Zeller, A.; Rychlik, M. Impact of estragole and other odorants on the flavour of anise and tarragon. Flavour Frag. J. 2007, 22, 105-113. [CrossRef]

28. Figiel, A.; Szumny, A.; Gutiérrez-Ortíz, A.; Carbonell-Barrachina, Á.A. Composition of oregano essential oil (Origanum vulgare) as affected by drying method. J. Food Eng. 2010, 98, 240-247. [CrossRef]

29. Cuvelier, M.E.; Richard, H.; Berset, C. Antioxidative activity and phenolic composition of pilot-plant and commercial extracts of sage and rosemary. J. Am. Oil Chem. Soc. 1996, 73, 645-652. [CrossRef]

30. Cai, Y.; Luo, Q.; Sun, M.; Corke, H. Antioxidant activity and phenolic compounds of 112 traditional Chinese medicinal plants associated with anticancer. Life Sci. 2004, 74, 2157-2184. [CrossRef]

31. Štěrbová, D.; Matějíček, D.; Vlček, J.; Kubáň, V. Combined microwave-assisted isolation and solid-phase purification procedures prior to the chromatographic determination of phenolic compounds in plant materials. Anal. Chim. Acta 2004, 513, 435-444.

32. Rodríguez-Solana, R.; Salgado, J.M.; Domínguez, J.M.; Cortés-Diéguez, S. Phenolic compounds and aroma-impact odorants in herb liqueurs elaborated by maceration of aromatic and medicinal plants in grape marc distillates. J. Inst. Brew. 2016, 122, 653-660. [CrossRef]

33. Diéguez, S.; de la Peña, M.L.; Gómez, E. Volatile composition and sensory characters of commercial Galician orujo spirits. J. Agric. Food Chem. 2005, 53, 6759-6765. [CrossRef] [PubMed]

34. Rodríguez-Solana, R.; Salgado, J.M.; Domínguez, J.M.; Cortés-Diéguez, S. Comparison of soxhlet, accelerated solvent and supercritical fluid extraction techniques for volatile (GC-MS and GC-FID) and phenolic compounds (HPLC-ESI/MS/MS) from lamiaceae species. Phytochem. Anal. 2015, 26, 61-71. [CrossRef] [PubMed]

35. Galicia Council. Official Diary from Galicia Council Regulation 2012/1/16 on the definition, description and presentation of Geographical Indications Orujo, spirits herbs, herbal liqueur and coffee liqueur from Galicia. Galician Off. Pap. 2012, 10, 2516-2546.

36. Rodríguez-Solana, R.; Salgado, J.M.; Domínguez, J.M. Cortés-Diéguez, S. Optimization of the process of aromatic and medicinal plant maceration in grape marc distillates to obtain herbal liqueurs and spirits. J. Sci. Food Agric. 2016, 96, 4760-4771. [CrossRef] [PubMed]

37. Cortés, S.; Fernández, A.; Otero, C.; Salgado, I. Application of sensory descriptive analysis to complete the current official card of the Galician Orujo spirits. J. Sens. Stud. 2009, 24, 317-331. [CrossRef]

38. Suwonsichon, S. Review: The Importance of Sensory Lexicons for Research and Development of Food Products. Foods 2019, 8, 27. [CrossRef] 
39. Elgaard, L.; Mielby, L.A.; Heymann, H.; Byme, D.V. Effect of product involvement on panel's vocabulary generation, attribute identification, and sample configurations in beer. Foods 2019, 8, 488. [CrossRef]

40. Ickes, C.M.; Cadwallader, K.R. Characterization of sensory differences in mixing and premium rums through the use of descriptive sensory analysis. J. Food Sci. 2017, 82, 2679-2689. [CrossRef]

41. Lesschaeve, I.; Noble, A. Polyphenols: Factors influencing their sensory properties and their effects on food and beverage preferences 1-3. Am. J. Clin. Nutr. 2005, 81, 330S-333S. [CrossRef]

42. Drake, M.; Civille, G. Flavor lexicons. Comprehensive reviews. Food Sci. Food Saf. 2003, 2, 33-40. [CrossRef]

43. Vilanova, M.; Vilariño, F. Influence of geographic origin on aromatic descriptors of Spanish albariño wine. Flavour Frag. J. 2006, 21, 373-378. [CrossRef]

44. Donnell, E.M.C.; Hulin-Bertraud, S.; Sheehan, E.M.; Delahunty, C.M. Development and learning process of a sensory vocabulary for the odor evaluation of selected distilled beverages using descriptive analysis. J. Sens. Stud. 2007, 16, 425-445. [CrossRef]

45. Caldeira, I.; Lopes, D.; Delgado, T.; Canas, S.; Anjos, O. Development of blueberry liquor. Influence of distillate, swe-tener and fruity quantity. J. Sci. Food Agric. 2018, 98, 1088-1094. [CrossRef]

46. Nykänen, L.; Nykänen, L. Distilled Beverages. In Volatile Compounds in Foods and Beverages; Maarse, H., Ed.; Marcel Dekker: New York, YN, USA, 1991; pp. 547-580.

47. Reinoso-Carvalho, F.; Dakduk, S.; Wagemans, J.; Spence, C. Dark vs. light drinks: The influence of visual appearance on the consumer's experience of beer. Food Qual. Pref. 2019, 74, 21-29. [CrossRef]

48. Gonzalez, G.M.; Lawrence, C.; Perez, R.A. Development of a structured sensory analysis honey: Application to artisanal honeys Madrid. Food Sci. Tech. Int. 2010, 16, 19-29. [CrossRef]

49. Navarro-Martínez, P.; Noguera-Artiaga, L.; Sánchez-Bravo, P.; Rosas-Burgos, P.; Rosas-Burgos, E.; Pérez-López, A.; Carbonell-Barrachina, A.A. Development and characterization of liquors prepared with an underutilized citrus by-product, the peel. Eur. Food Res. Tecnol. 2019, 245, 41-50. [CrossRef]

50. Senica, M.; Mikulic-Petkovsek, M. Changes in beneficial bioactive compounds in eight tradicional herbal liqueurs during a one-month maceration. J. Sci. Food Agric. 2020, 100, 343-353. [CrossRef]

51. Petrovic, M.; Vukosavljevic, P.; Durovic, S.; Antic, M.; Gorjanovic, S. New herbal bitter liqueur with high antioxidant activity and lower sugar content: Innovative approach to liqueurs formulations. J. Food Sci. Technol. 2019, 56, 4465-4473. [CrossRef]

52. Castañeda-Olivares, F.; Pless, R.C.; González-Jasso, E. Effect of Light and sweeteners on color in an Amaretto-type liqueur. J. Food Sci. 2010, 75, C766-C773. [CrossRef]

53. Shen, S.-C.; Tseng, K.-C.; Chao, F.-I.; Wu, J.S.-B. Color quality of rose liqueur. J. Food Qual. 2007, 30, $202-217$. [CrossRef]

54. Chambers, E.; Sanchez, K.; Phan, U.X.T.; Miller, R.; Civille, G.V.; Donfrancesco, B. Development of a "living" lexicon for descriptive sensory analysis of brewed coffee. J. Sens. Stud. 2016, 31, 465-480. [CrossRef]

55. Galán-Soldevilla, H.; Ruiz-Pérez-Cacho, M.P.; Serrano Jiménez, S.; Jodral Villarejo, M.; Bentabol Manzanares, A. Development of a preliminary sensory lexicon for floral Honey. Food Qual. Pref. 2005, 16, 71-77. [CrossRef]

(C) 2020 by the authors. Licensee MDPI, Basel, Switzerland. This article is an open access article distributed under the terms and conditions of the Creative Commons Attribution (CC BY) license (http://creativecommons.org/licenses/by/4.0/). 